Article

\title{
Energy, Momentum, Mass and Velocity of a Moving Body
}

\section{Sergey G. Fedosin}

PO box 614088, Sviazeva str. 22-79, Perm, Perm Krai, Russia; fedosin@hotmail.com

\begin{abstract}
In the weak-field approximation of covariant theory of gravitation the problem of $4 / 3$ is formulated for internal and external gravitational fields of a body in the form of a ball. The dependence of the energy and the mass of the moving substance on the energy of field accompanying the substance, as well as the dependence on the characteristic size of the volume occupied by the substance are described. Additives in the energy and the momentum of the body, defined by energy and momentum of the gravitational and electromagnetic fields associated with the body are explicitly calculated. The conclusion is made that the energy and the mass of the body can be described by the energy of ordinary and strong gravitation, and through the energies of electromagnetic fields of particles that compose the body.
\end{abstract}

Keywords: energy; momentum; theory of relativity; gravitation; field potentials

\section{Introduction}

In relativistic mechanics, there are standard formulas for the dependence of total energy and momentum of a particle with the mass $m$ on its velocity $V$ :

$$
E=\frac{m c^{2}}{\sqrt{1-V^{2} / c^{2}}}, \quad \boldsymbol{p}=\frac{m \boldsymbol{V}}{\sqrt{1-V^{2} / c^{2}}} .
$$

If the energy $E$ and momentum $\boldsymbol{p}$ in (1) are known the mass and the velocity of the particle can be calculated:

$$
m=\frac{1}{c^{2}} \sqrt{E^{2}-p^{2} c^{2}}, \quad \quad \boldsymbol{V}=\frac{\boldsymbol{p} c^{2}}{E}
$$

In (1) and (2) the speed of light $c$ is included. For a particle in rest velocity and momentum are zero, and the total energy of the particle equals the rest energy:

$$
E_{0}=m c^{2} \text {. }
$$

Equation (3) reflects the principle of proportionality of mass and energy. In elementary particle physics the energy and the momentum are usually measured parameters, and the mass and the velocity are found from (2) and are secondary parameters.

Now, we shall suppose that the measured parameters are the energy and the velocity of the particle. In this case, from (1) we can calculate the mass and momentum:

$$
m=\frac{E \sqrt{1-V^{2} / c^{2}}}{c^{2}}, \quad \quad \boldsymbol{p}=\frac{E \boldsymbol{V}}{c^{2}} .
$$

The case is also possible when the measured parameters are the momentum and the velocity of the particle and the calculated quantities are the mass and energy: 


$$
m=\frac{p \sqrt{1-V^{2} / c^{2}}}{V}, \quad E=\frac{p c^{2}}{V} .
$$

If the particle velocity $V$ is given, then the mass can be found either through the energy according to (4), or through the momentum according to (5), in both cases, the mass should be the same.

There are also two possible combinations of parameters when the energy and the mass, or the momentum and the mass are known. This allows us calculating the absolute value of the momentum and the velocity, or the energy and the velocity, respectively:

$$
\begin{array}{ll}
p=\frac{1}{c} \sqrt{E^{2}-m^{2} c^{4}}, & V=\frac{c}{E} \sqrt{E^{2}-m^{2} c^{4}}, \\
E=c \sqrt{p^{2}+m^{2} c^{2}}, & \boldsymbol{V}=\frac{\boldsymbol{p} c}{\sqrt{p^{2}+m^{2} c^{2}}} .
\end{array}
$$

From the above formulas it is not clear whether they contain the energy and the momentum of fields, which are inherent in the particles and the test bodies. In particular, the test bodies always have their proper gravitational field and can also carry an electrical charge and the corresponding electromagnetic field. In general theory of relativity (GTR) it is considered that relativistic energy and mass of a body decrease due to the contribution of the gravitational energy. Although in GTR there is no unique definition of the gravitational energy and its contribution to the total energy [1], in the weak-field approximation the following is assumed [2]:

$$
E_{g}=E+U, \quad M_{g}=\frac{E_{g}}{c^{2}} .
$$

where $E_{g}$ is the relativistic energy in the gravitational field, $E$ is the energy in the absence of the field, $U$ is the potential gravitational energy of the body.

Since the energy $U$ is negative, then according to GTR the mass of the body $M_{g}$ should decrease with increasing of the field.

The main purpose of this paper is to incorporate explicitly in the relativistic formulas for the energy and the momentum the additives, resulting from the energy and the momentum of fields associated with the test bodies. All subsequent calculations will be made in the framework of the covariant theory of gravitation (CTG) [3]. We will apply the weak-field approximation, when CTG is transformed into the Lorentz-invariant theory of gravitation (LITG), and it becomes possible to compare our results with the formulas of GTR (6).

\section{External gravitational field. Problem of $4 / 3$}

We shall assume that (1) - (5) are valid for a small particle and energy of its proper gravitational field is taken into account through its mass. If there are a lot of particles in the volume of the body, then their interaction energy leads to a significant contribution of the field energy into the total energy of the body. In order to simplify calculations, we shall further consider the case of a weak field. In a weak field can be assumed that either the spacetime metric differs little from the metric of Minkowski spacetime, or the gravitational effects of time dilation and reducing of size are considerably smaller than the similar effects due to the motion of the body. In this approximation the general theory of relativity turns to gravitomagnetism, the covariant theory of gravitation turns to the Lorentzinvariant theory of gravitation (LITG).

Gravitational potentials of the substance unit of point size, located at $t=0$ in a point $\left(x_{0}, y_{0}, z_{0}\right)$ of space and moving along the axis $O X$ at a constant speed $V$, according to [4] are as follows: 


$$
d \psi=-\frac{\gamma d M}{\sqrt{1-V^{2} / c^{2}} \sqrt{\frac{\left(x-x_{0}-V t\right)^{2}}{1-V^{2} / c^{2}}+\left(y-y_{0}\right)^{2}+\left(z-z_{0}\right)^{2}}}, \quad d \boldsymbol{D}=\frac{d \psi \boldsymbol{V}}{c_{g}^{2}},
$$

here $d \psi$ - the scalar potential,

$\gamma$ - the gravitational constant,

$d M$ - the mass of substance unit,

$c_{g}$ - the propagation speed of gravitation, which further for simplification of the calculations, we shall assume equal to the speed of light $c$,

$(x, y, z)$ - the coordinates of the point at which the potential is determined at the time $t$, $d \boldsymbol{D}$ - vector potential.

According to (7), the gravitational potential $d \psi$ at the time $t$ of the point mass $d M$ moving along the axis $O X$ depends on the initial position $\left(x_{0}, y_{0}, z_{0}\right)$ of this mass at $t=0$. After integration of (7) over all point masses inside the ball on the basis of the principle of superposition, the standard formulas are obtained for the potentials of gravitational field around the moving ball, with retardation of the gravitational interaction taken into account:

$$
\psi=-\frac{\gamma M}{\sqrt{(x-V t)^{2}+\left(1-V^{2} / c^{2}\right)\left(y^{2}+z^{2}\right)}}, \quad \boldsymbol{D}=\frac{\psi \boldsymbol{V}}{c^{2}},
$$

where $\psi-$ the scalar potential of the moving ball,

$M$ - the mass of the ball,

$(x, y, z)$ - the coordinates of the point at which the potential is determined at the time $t$ (on the condition that the center of the ball at $t=0$ was in the origin of coordinate system),

D - the vector potential.

In (8) it is assumed that the ball is moving along the axis $O X$ at a constant speed $V$, so that $D_{x}=\frac{\psi V}{c^{2}}, D_{y}=0, D_{z}=0$. With the help of the field potentials we can calculate the field strengths around the ball by the formulas [5]:

$$
\boldsymbol{G}=-\nabla \psi-\frac{\partial \boldsymbol{D}}{\partial t}, \quad \boldsymbol{\Omega}=\nabla \times \boldsymbol{D}
$$

where $\boldsymbol{G}$ is the gravitational acceleration,

$\boldsymbol{\Omega}$ - the gravitational torsion in LITG (gravitomagnetic field in gravitomagnetism).

In view of (8) and (9) we find:

$$
\begin{gathered}
G_{x}=-\frac{\gamma M(x-V t)\left(1-V^{2} / c^{2}\right)}{\sqrt{\left[(x-V t)^{2}+\left(1-V^{2} / c^{2}\right)\left(y^{2}+z^{2}\right)\right]^{3}}}, \\
G_{y}=-\frac{\gamma M y\left(1-V^{2} / c^{2}\right)}{\sqrt{\left[(x-V t)^{2}+\left(1-V^{2} / c^{2}\right)\left(y^{2}+z^{2}\right)\right]^{3}}}, \\
G_{z}=-\frac{\gamma M z\left(1-V^{2} / c^{2}\right)}{\sqrt{\left[(x-V t)^{2}+\left(1-V^{2} / c^{2}\right)\left(y^{2}+z^{2}\right)\right]^{3}}},
\end{gathered}
$$




$$
\begin{gathered}
\Omega_{y}=\frac{\gamma M z V\left(1-V^{2} / c^{2}\right)}{c^{2} \sqrt{\left[(x-V t)^{2}+\left(1-V^{2} / c^{2}\right)\left(y^{2}+z^{2}\right)\right]^{3}}} \\
\Omega_{z}=-\frac{\gamma M y V\left(1-V^{2} / c^{2}\right)}{c^{2} \sqrt{\left[(x-V t)^{2}+\left(1-V^{2} / c^{2}\right)\left(y^{2}+z^{2}\right)\right]^{3}}}
\end{gathered}
$$

The energy density of the gravitational field is determined by the formula [5]:

$$
u=-\frac{1}{8 \pi \gamma}\left(G^{2}+c^{2} \Omega^{2}\right)=-\frac{\gamma M^{2}\left(1-V^{2} / c^{2}\right)\left[(x-V t)^{2}+\left(1+V^{2} / c^{2}\right)\left(y^{2}+z^{2}\right)\right]}{8 \pi\left[(x-V t)^{2}+\left(1-V^{2} / c^{2}\right)\left(y^{2}+z^{2}\right)\right]^{3}} .
$$

The total energy of the field outside the ball at a constant velocity should not depend on time. So it is possible to integrate the energy density of the field (11) over the external space volume at $t=0$. For this purpose we shall introduce new coordinates:

$$
x=\sqrt{1-V^{2} / c^{2}} r \cos \theta, \quad y=r \sin \theta \cos \varphi, \quad z=r \sin \theta \sin \varphi .
$$

The volume element is determined by the formula $d \Upsilon=J d r d \theta d \varphi$, where:

$$
J=\frac{\partial(x, y, z)}{\partial(r, \theta, \varphi)}=\left|\begin{array}{lll}
\frac{\partial x}{\partial r} & \frac{\partial x}{\partial \theta} & \frac{\partial x}{\partial \varphi} \\
\frac{\partial y}{\partial r} & \frac{\partial y}{\partial \theta} & \frac{\partial y}{\partial \varphi} \\
\frac{\partial z}{\partial r} & \frac{\partial z}{\partial \theta} & \frac{\partial z}{\partial \varphi}
\end{array}\right|
$$

It follows that $d \Upsilon=r^{2} \sin \theta \sqrt{1-V^{2} / c^{2}} d r d \theta d \varphi$.The integral over the space of the energy density (11) will equal:

$$
U_{b}=\int u d \Upsilon=-\frac{\gamma M^{2}}{8 \pi c^{2} \sqrt{1-V^{2} / c^{2}}} \int \frac{\left[c^{2}+V^{2}\left(\sin ^{2} \theta-\cos ^{2} \theta\right)\right] \sin \theta d r d \theta d \varphi}{r^{2}} .
$$

We shall take into account that due to the Lorentz contraction during the motion along the axis $O X$ the ball must be as an ellipsoid, the surface equation of which at $t=0$ is the following:

$$
\frac{x^{2}}{1-V^{2} / c^{2}}+y^{2}+z^{2}=R^{2}
$$

After substituting (12) in (14), it becomes apparent that the radius $r$ at the integration in (13) must change from $R$ to $\infty$, and the angles $\theta$ and $\varphi$ change the same way as in spherical coordinates (from 0 to $\pi$ for the angle $\theta$, and from 0 to $2 \pi$ for the angle $\varphi$ ). For the energy of the gravitational field outside the moving ball we find:

$$
U_{b}=-\frac{\gamma M^{2}\left(1+V^{2} / 3 c^{2}\right)}{2 R \sqrt{1-V^{2} / c^{2}}}=\frac{U_{b 0}\left(1+V^{2} / 3 c^{2}\right)}{\sqrt{1-V^{2} / c^{2}}}
$$

where $U_{b 0}=-\frac{\gamma M^{2}}{2 R}$ is the field energy around the stationary ball.

We shall assume that the formula (4) for connection of the mass and the energy of the particle is also valid for the gravitational field. Then we can introduce the effective mass of the field related to the energy: 


$$
m_{g b}=\frac{U_{b} \sqrt{1-V^{2} / c^{2}}}{c^{2}}=\frac{U_{b 0}\left(1+V^{2} / 3 c^{2}\right)}{c^{2}} .
$$

We shall now consider the momentum density of the gravitational field:

$$
g=\frac{S}{c^{2}},
$$

where $\boldsymbol{S}=-\frac{c^{2}}{4 \pi \gamma}[\boldsymbol{G} \times \boldsymbol{\Omega}]$ is the vector of energy flux density of the gravitational field [5].

Substituting in (17) the components of the field (10), we find:

$$
\begin{gathered}
g_{x}=-\frac{\gamma M^{2}\left(1-V^{2} / c^{2}\right)^{2}\left(y^{2}+z^{2}\right) V}{4 \pi c^{2}\left[(x-V t)^{2}+\left(1-V^{2} / c^{2}\right)\left(y^{2}+z^{2}\right)\right]^{3}}, \\
g_{y}=\frac{\gamma M^{2}\left(1-V^{2} / c^{2}\right)^{2}(x-V t) y V}{4 \pi c^{2}\left[(x-V t)^{2}+\left(1-V^{2} / c^{2}\right)\left(y^{2}+z^{2}\right)\right]^{3}}, \\
g_{z}=\frac{\gamma M^{2}\left(1-V^{2} / c^{2}\right)^{2}(x-V t) z V}{4 \pi c^{2}\left[(x-V t)^{2}+\left(1-V^{2} / c^{2}\right)\left(y^{2}+z^{2}\right)\right]^{3}} .
\end{gathered}
$$

We can see that the components of the momentum density of gravitational field (18) look the same as if a liquid flowed around the ball from the axis $O X$, carrying similar density of the momentum - liquid spreads out to the sides when meeting with the ball and merges once again on the opposite side of the ball. Integrating the components of the momentum density of the gravitational field (18) by volume outside the moving ball at $t=0$ as in (13), we obtain:

$$
\begin{array}{cc}
\Gamma_{x}=\int g_{x} d \Upsilon=-\frac{\gamma M^{2} V}{4 \pi c^{2} \sqrt{1-V^{2} / c^{2}}} \int \frac{\sin ^{3} \theta d r d \theta d \varphi}{r^{2}}=-\frac{2 \gamma M^{2} V}{3 R c^{2} \sqrt{1-V^{2} / c^{2}}} . \\
\Gamma_{y}=\int g_{y} d \Upsilon=0, & \Gamma_{z}=\int g_{z} d \Upsilon=0 .
\end{array}
$$

In (19) the total momentum of the field has only the component along the axis $O X$. By analogy with (5) the coefficient before the velocity $V$ in (19) can be interpreted as the effective mass of the external gravitational field moving with the ball:

$$
m_{p b}=\frac{\Gamma_{x} \sqrt{1-V^{2} / c^{2}}}{V}=-\frac{2 \gamma M^{2}}{3 R c^{2}}=\frac{4 U_{b 0}}{3 c^{2}},
$$

where $U_{b 0}=-\frac{\gamma M^{2}}{2 R}$ is the energy of the external static field of the ball at rest.

Comparing (20) and (16) gives:

$$
m_{g b}=\frac{3\left(1+V^{2} / 3 c^{2}\right) m_{p b}}{4} .
$$

The discrepancy between the masses $m_{g b}$ and $m_{p b}$ in (21) is the essence of the so-called problem of $4 / 3$, according to which the mass $m_{p b}$ of the field, which is calculated through the momentum of the field, at low speeds is approximately $4 / 3$ larger than the mass $m_{g b}$ of the field, found through the field energy. A characteristic feature of the fundamental fields, which include the gravitational and electromagnetic fields, is the similarity of their equations for the potentials and the 
6 of 17

field strengths. The problem of $4 / 3$ is known for a long time for the mass of electromagnetic field of a moving charge. Joseph John Thomson, George Francis FitzGerald, Oliver Heaviside [6], George Frederick Charles Searle and many others wrote about it in the late 19-th century. We also discussed this question previously with respect to the gravitational field of a moving ball [7]. Now we present an exact solution of the problem, not limited to the approximation of small velocities.

\section{The gravitational field inside a moving ball}

According to [4] for a ball with the density of substance $\bar{\rho}$ (measured in the comoving frame), which is moving along the axis $O X$, the potentials inside the ball (denoted by subscript $i$ ) depend on time and are as follows:

$$
\psi_{i}=-\frac{2 \pi \gamma \bar{\rho}}{\sqrt{1-V^{2} / c^{2}}}\left[R^{2}-\frac{1}{3}\left(\frac{(x-V t)^{2}}{1-V^{2} / c^{2}}+y^{2}+z^{2}\right)\right], \quad \boldsymbol{D}_{i}=\frac{\psi_{i} \boldsymbol{V}}{c^{2}}
$$

In view of (9) we can calculate the internal strengths of field:

$$
\begin{gathered}
G_{x i}=-\frac{4 \pi \gamma \bar{\rho}(x-V t)}{3 \sqrt{1-V^{2} / c^{2}}}, \quad G_{y i}=-\frac{4 \pi \gamma \bar{\rho} y}{3 \sqrt{1-V^{2} / c^{2}}}, \quad G_{z i}=-\frac{4 \pi \gamma \bar{\rho} z}{3 \sqrt{1-V^{2} / c^{2}}}, \\
\Omega_{x i}=0, \Omega_{y i}=\frac{4 \pi \gamma \bar{\rho} z V}{3 c^{2} \sqrt{1-V^{2} / c^{2}}}, \quad \Omega_{z i}=-\frac{4 \pi \gamma \bar{\rho} y V}{3 c^{2} \sqrt{1-V^{2} / c^{2}}}
\end{gathered}
$$

Similarly to (11) for the energy density of the field we find:

$$
u_{i}=-\frac{1}{8 \pi \gamma}\left(G_{i}^{2}+c^{2} \Omega_{i}^{2}\right)=-\frac{2 \pi \gamma \bar{\rho}^{2}\left[(x-V t)^{2}+\left(1+V^{2} / c^{2}\right)\left(y^{2}+z^{2}\right)\right]}{9\left(1-V^{2} / c^{2}\right)} .
$$

According to (24) the minimum energy density inside a moving ball is achieved on its surface, and in the center at $t=0$ it is zero.

The integral of (24) by volume of the ball at $t=0$ in coordinates (12) with the volume element $d \Upsilon=r^{2} \sin \theta \sqrt{1-V^{2} / c^{2}} d r d \theta d \varphi$ equals:

$$
U_{i}=\int u_{i} d \Upsilon=-\frac{2 \pi \gamma \bar{\rho}^{2}}{9 c^{2} \sqrt{1-V^{2} / c^{2}}} \int\left[c^{2}+V^{2}\left(\sin ^{2} \theta-\cos ^{2} \theta\right)\right] r^{4} \sin \theta d r d \theta d \varphi .
$$

The moving ball looks like an ellipsoid with the surface equation (14), and in the coordinates (12) the radius in the integration in (25) varies from 0 to $R$. With this in mind for the energy of the gravitational field inside the moving ball, we have:

$$
U_{i}=-\frac{\gamma M^{2}\left(1+V^{2} / 3 c^{2}\right)}{10 R \sqrt{1-V^{2} / c^{2}}}=\frac{U_{i 0}\left(1+V^{2} / 3 c^{2}\right)}{\sqrt{1-V^{2} / c^{2}}}
$$

where $U_{i 0}=-\frac{\gamma M^{2}}{10 R}$ is the field energy inside a stationary ball with radius $R$.

The effective mass of the field associated with energy is obtained similarly (4):

$$
m_{g i}=\frac{U_{i} \sqrt{1-V^{2} / c^{2}}}{c^{2}}=\frac{U_{i 0}\left(1+V^{2} / 3 c^{2}\right)}{c^{2}} .
$$

Substituting in (17) the components of the field strengths (23), we find the components of the vector of momentum density of the gravitational field: 


$$
g_{x i}=-\frac{4 \pi \gamma \bar{\rho}^{2}\left(y^{2}+z^{2}\right) V}{9 c^{2}\left(1-V^{2} / c^{2}\right)}, \quad g_{y i}=\frac{4 \pi \gamma \bar{\rho}^{2}(x-V t) y V}{9 c^{2}\left(1-V^{2} / c^{2}\right)}, \quad g_{z i}=\frac{4 \pi \gamma \bar{\rho}^{2}(x-V t) z V}{9 c^{2}\left(1-V^{2} / c^{2}\right)}
$$

The vector connecting the origin of coordinate system and center of the ball depends on the time and has the components $(V t, 0,0)$. From this in the point, coinciding with the center of the ball, the components of the vector of the momentum density of the gravitational field are always zero. At $t=0$ the center of the ball passes through the origin of the coordinate system, and at the time from (28) it follows that the maximum density of the field momentum $g_{\max }=-\frac{4 \pi \gamma \bar{\rho}^{2} R^{2} V}{9 c^{2}\left(1-V^{2} / c^{2}\right)}=-\frac{\gamma M^{2} V}{4 \pi R^{4} c^{2}\left(1-V^{2} / c^{2}\right)}$ is achieved on the surface of the ball on the circle of radius $R$ in the plane $Y O Z$, which is perpendicular to the line $O X$ of the ball's motion. The same follows from (18).

We can integrate the components of the momentum density of gravitational field (28) over the volume inside the moving ball at $t=0$ in the coordinates (12) similar to (19):

$$
\begin{array}{cc}
\Gamma_{x i}=\int g_{x i} d \Upsilon=-\frac{4 \pi \gamma \bar{\rho}^{2} V}{9 c^{2} \sqrt{1-V^{2} / c^{2}}} \int r^{4} \sin ^{3} \theta d r d \theta d \varphi=-\frac{2 \gamma M^{2} V}{15 R c^{2} \sqrt{1-V^{2} / c^{2}}} . \\
\Gamma_{y i}=\int g_{y i} d \Upsilon=0, & \Gamma_{z i}=\int g_{z i} d \Upsilon=0 .
\end{array}
$$

As in (19), the total momentum of the field (29) has only the component along the axis $O X$. By analogy with (5) the coefficient before the velocity $V$ in (29) is interpreted as the effective mass of the gravitational field inside the ball:

$$
m_{p i}=\frac{\Gamma_{x i} \sqrt{1-V^{2} / c^{2}}}{V}=-\frac{2 \gamma M^{2}}{15 R c^{2}}=\frac{4 U_{i 0}}{3 c^{2}}
$$

where $U_{i 0}=-\frac{\gamma M^{2}}{10 R}$ is the field energy inside a stationary ball.

Comparing (27) and (30) gives:

$$
m_{g i}=\frac{3\left(1+V^{2} / 3 c^{2}\right) m_{p i}}{4}
$$

Connection (31) between the masses of the field inside the ball is the same as in (21) for the masses of the external field, so the problem of $4 / 3$ exists inside the ball too.

\section{The contribution of gravitational field in energy and momentum of a moving body}

We shall try to include in equation (1) the relations found above for the energy and the momentum of the gravitational field of a moving test body in the form of a ball. We shall suppose that in static case instead of (3) there is the following relation:

$$
E_{0}=E_{0 s}-U_{0}
$$

where $U_{0}=U_{b 0}+U_{i 0}=-\frac{3 \gamma M^{2}}{5 R}-$ the total energy of static gravitational field inside and outside the ball with uniform density of the substance, 
$E_{0 s}$ - the rest energy of the substance, found in such a way that it does not depend on the energy of the gravitational field. To determine the energy $E_{0 s}$ the body's substance should be divided into pieces and spread to infinity.

Choosing the minus sign before $U_{0}$ in (32) will be substantiated in the last section. We shall continue to analyze the well-known thought experiment. We shall assume that the substance of the ball is composed of matter and antimatter, which at some time begin to annihilate and emit photons. We shall suppose that the photons fly in opposite directions along the axis $O X$ in the number of $N$ in each direction, so that eventually the entire mass of the ball turns into electromagnetic radiation. In the course of emission because of the equality of all momentums of photons, and the symmetry of the radiation along the axis $O X$ the ball remains stationary. In order that the process does not depend on the radius of the ball, we shall assume that the radius is constant irrespective of changes in mass. The energy $E_{0}$ of the ball (32) should be transformed into the energy of photons:

$$
E_{0}=2 N h v_{0},
$$

where $h$ - Planck constant,

$v_{0}-$ the frequency of photons.

We shall consider the same situation in the frame of reference $K$, in which the ball moves at constant velocity $V$ along the axis $O X$ and at $t=0$ it is in the origin of coordinate system. We believe that the velocity of the ball does not change, despite the emission of photons. In the frame $K$ the frequency of the photons will depend on whether they are flying along the axis $O X$ or in the opposite direction. Taking into account the relativistic Doppler effect and (32), for the photon energy instead of (33) we shall obtain:

$$
\begin{aligned}
E=N h v_{1}+N h v_{2}= & \frac{N h v_{0} \sqrt{1-V^{2} / c^{2}}}{1-V / c}+\frac{N h v_{0} \sqrt{1-V^{2} / c^{2}}}{1+V / c}= \\
& =\frac{2 N h v_{0}}{\sqrt{1-V^{2} / c^{2}}}=\frac{E_{0}}{\sqrt{1-V^{2} / c^{2}}}=\frac{E_{0 s}-U_{0}}{\sqrt{1-V^{2} / c^{2}}} .
\end{aligned}
$$

On the other hand, the total energy of the gravitational field inside and outside the ball, taking into account (15) and (26) equals:

$$
U=U_{b}+U_{i}=-\frac{3 \gamma M^{2}\left(1+V^{2} / 3 c^{2}\right)}{5 R \sqrt{1-V^{2} / c^{2}}}=\frac{U_{0}\left(1+V^{2} / 3 c^{2}\right)}{\sqrt{1-V^{2} / c^{2}}} .
$$

For the energy of substance and field of the moving ball, we have:

$$
E=E_{s}-U=E_{s}-\frac{U_{0}\left(1+V^{2} / 3 c^{2}\right)}{\sqrt{1-V^{2} / c^{2}}} .
$$

From (34) and (36) it follows:

$$
E_{s}=\frac{E_{0 s}}{\sqrt{1-V^{2} / c^{2}}}+\frac{U_{0} V^{2}}{3 c^{2} \sqrt{1-V^{2} / c^{2}}} .
$$


Since the energy of the static field is negative: $U_{0}=-\frac{3 \gamma M^{2}}{5 R}$, then in (37) in the energy $E_{s}$ of substance of the moving ball the negative additive from field energy will appear, and the energy $E_{0 s}$ does not depend on $U_{0}$.

We shall consider now the law of conservation of momentum. Before emission of the photons the momentum of the moving ball consists of the momentum of the ball's substance and the momentum of the gravitational field, and taking into account (19) for the field momentum outside the ball, and (29) for the momentum of the field inside the ball, the total momentum of the field is:

$$
P_{f}=-\frac{4 \gamma M^{2} V}{5 R c^{2} \sqrt{1-V^{2} / c^{2}}}
$$

Then for the momentum of a moving ball we can write down:

$$
P=P_{s}+P_{f}=M_{V} V-\frac{4 \gamma M^{2} V}{5 R c^{2} \sqrt{1-V^{2} / c^{2}}}=M_{V} V+\frac{4 U_{0} V}{3 c^{2} \sqrt{1-V^{2} / c^{2}}}
$$

where $M_{V}$ is the mass of the substance of the ball as a function of the velocity $V$.

After the photon emission the whole momentum of the ball and its gravitational field turns into the momentum of photons:

$$
P=\frac{N h v_{1}}{c}-\frac{N h v_{2}}{c}=\frac{2 N h v_{0} V}{c^{2} \sqrt{1-V^{2} / c^{2}}}=\frac{E_{0} V}{c^{2} \sqrt{1-V^{2} / c^{2}}}=\frac{\left(E_{0 s}-U_{0}\right) V}{c^{2} \sqrt{1-V^{2} / c^{2}}},
$$

where $E_{0}=E_{0 s}-U_{0}$ is the energy (32) of the ball at rest, equal to the difference of the rest energy $E_{0 s}$ of the substance and the energy of the gravitational field $U_{0}$; also $E_{0}$ is the energy of photons according to (33). From comparing (38) and (39) it follows:

$$
E_{0 s}=M_{V} c^{2} \sqrt{1-V^{2} / c^{2}}+\frac{7 U_{0}}{3} .
$$

We shall suppose that the mass of the moving substance of the ball is described by the formula: $M_{V}=\frac{\alpha M}{\sqrt{1-V^{2} / c^{2}}}$, where $M$ is the observed mass of the body at rest, and $\alpha$ is a function. Here we assume that the observed mass $M$ of the body, and that the mass through which the energy $U_{0}$ and the momentum $P_{f}$ of the gravitational field are determined, are the same mass. Then instead of (40) we shall have:

$$
E_{0 s}=\alpha M c^{2}+\frac{7 U_{0}}{3}
$$

But the energy $E_{0 s}$ of substance at rest should not depend on the speed, as well as on the field energy $U_{0}$ of a stationary ball according to (32). Therefore, in (41) we should have $\alpha=\beta-\frac{7 U_{0}}{3 M c^{2}}$ , which in view of (40) implies the following:

$$
E_{0 s}=\beta M c^{2}=m^{\prime} c^{2}, \quad M_{V}=\frac{m^{\prime}}{\sqrt{1-V^{2} / c^{2}}}-\frac{7 U_{0}}{3 c^{2} \sqrt{1-V^{2} / c^{2}}},
$$

where the mass $m^{\prime}$ sets the rest energy of substance $E_{0 s}$ in (32). 
We shall substitute $E_{0 s}$ from (42) in (37):

$$
E_{s}=\frac{m^{\prime} c^{2}}{\sqrt{1-V^{2} / c^{2}}}+\frac{U_{0} V^{2}}{3 c^{2} \sqrt{1-V^{2} / c^{2}}} .
$$

From (43) it follows that at $V=0$ the energy of the substance does not include the field energy, but during moving in the energy $E_{s}$ of the substance an additive appears, related with the energy $U_{0}$ of the field. The field energy $U_{0}$ also makes contribution to the mass $M_{V}$ of the moving substance in (42). The total energy (36) of the moving substance and the field in view of (43) will equal:

$$
E=\frac{m^{\prime} c^{2}-U_{0}}{\sqrt{1-V^{2} / c^{2}}}
$$

where in the case of uniform density of the ball substance $U_{0}=-\frac{3 \gamma M^{2}}{5 R}$.

Equation (44) implies that the energy of the body increases due to the contribution of negative gravitational energy $U_{0}$.

We shall now substitute $M_{V}$ from (42) in (38) or $E_{0 s}$ from (42) in (39). This gives the following:

$$
\boldsymbol{P}=\frac{\left(m^{\prime} c^{2}-U_{0}\right) \boldsymbol{V}}{c^{2} \sqrt{1-V^{2} / c^{2}}}
$$

Comparing (44) and (45) with (1) shows that taking into account the gravitational field the role of the total mass of the substance and the field is played by the quantity $M_{\Sigma}=m^{\prime}-U_{0} / c^{2}$. We believe that $M_{\Sigma}=M$, that is the total mass of the substance and the field, is nothing but the observed mass $M$, which also determines the gravitational field and the inert properties of the body.

If we know the energy $E$ in (44) and the momentum $\boldsymbol{P}$ (45), it follows from these relations that we can express the mass $M$ of the substance and the velocity $V$ of the body. In case of a uniform ball with radius $R$ in the calculation of the mass $M$ of the moving ball we can write down:

$$
M=\frac{1}{c^{2}} \sqrt{E^{2}-p^{2} c^{2}}=m^{\prime}+\frac{3 \gamma M^{2}}{5 R c^{2}}, \quad \boldsymbol{V}=\frac{\boldsymbol{P} c^{2}}{E} .
$$

According to (46), the mass of the body substance depends not only on the energy-momentum of the body, but also depends on the average body size due to the contribution of the gravitational field mass to the constant value of the mass $m^{\prime}$.

We shall note also that the problem of $4 / 3$ for the gravitational field (inequality of the mass of the field, found from the energy, and the mass of the field, calculated by the momentum of the field) was compensated by the dependence of the energy $E_{s}$ in (37) and the mass $M_{V}$ in (42) of the moving substance on the field energy $U_{0}$. As a result, the field energy $U_{0}$ in formulas (44) and (45) is included symmetrically in both the total energy and the total momentum of the body.

\section{Analysis of the components of mass and energy of the body}

Until now we have not specified of which components the mass $M$ of the substance of a body consists, and whether other energies except the energy of gravitational field contribute to it. For example, what shall happens if the body is heated? From the standpoint of kinetic theory, an increase 
of temperature leads first to an increase of the average velocity of the particles that makeup the body. In this case, according to (1) the average energy of each particle of the body would increase, and due to the additivity of energy the total energy $E_{\Sigma}$ of the body at rest should change. For the case of the substance and the gravitational field $E_{\Sigma}=m^{\prime} c^{2}-U_{0}=M c^{2}$, and for (44) - (45) we can write down the following:

$$
E=\frac{E_{\Sigma}}{\sqrt{1-V^{2} / c^{2}}}, \quad \quad \boldsymbol{P}=\frac{E_{\Sigma} \boldsymbol{V}}{c^{2} \sqrt{1-V^{2} / c^{2}}} .
$$

Heating of the body leads to the change of $E_{\Sigma}$ in (47), and the heat as a form of energy is distributed between the kinetic energy of substance and the energy $U_{0}$ of the gravitational field. The mass of the uniform ball can be determined through the mass $m^{\prime}$ from the relations:

$$
M c^{2}=m^{\prime} c^{2}-U_{0}=m^{\prime} c^{2}+\frac{3 \gamma M^{2}}{5 R}, \quad M=\frac{5 R c^{2}}{6 \gamma}\left(1-\sqrt{1-\frac{12 \gamma m^{\prime}}{5 R c^{2}}}\right) .
$$

Any interaction between particles of the body with each other or with the environment, which changes the energy of the particles, also changes the energy $E_{\Sigma}=M c^{2}$ of the body at rest. In accordance with (48) the mass $M$ of the substance of the ball depends not only on $m^{\prime}$, but also on the radius of the ball $R$.

Due to the similarity of equations of electromagnetic and gravitational fields, the energy $E_{\Sigma}$ must contain contribution from the total energy $W_{0}$ of the electromagnetic field of the body:

$$
E_{\Sigma}=m^{\prime} c^{2}-U_{0}-W_{0}
$$

For the uniformly charged by volume ball at rest with a charge $q$ the total energy of the electric field is:

$$
W_{0}=\frac{3 q^{2}}{20 \pi \varepsilon_{0} R}
$$

The energy of the magnetic field can make contribution to $W_{0}$, if the ball is magnetized or if there are electric currents. We assume that other forms of energy (e.g. heat) can change the body mass, but can not change the charge of the body, because it is necessary to transfer the charged particles to the body (or from the body). This is one of the differences between the electromagnetic and gravitational fields, in addition to the unipolarity of gravitational charges (which are the masses) and the bipolarity of electromagnetic charges.

The mass of the substance $m^{\prime}$ in (49) can be divided into two parts, one of which $m_{g}^{\prime}$ is the mass of the substance at zero temperature in Kelvin, and the other part $m_{k}^{\prime}$ is the additional mass from the internal kinetic energy $E_{k}$, which includes the kinetic energy of motion of atoms and molecules, and the energy of turbulent motion of substance flows [8]. If $V_{k}$ is the average velocity of particles in the body, then the following approximate relations would hold: $E_{k} \approx \frac{m^{\prime} V_{k}^{2}}{2}$, $m_{k}^{\prime}=\frac{E_{k}}{c^{2}} \approx \frac{m^{\prime} V_{k}^{2}}{2 c^{2}}$. As the energy of field, we include the energy $E_{k}$ in (49) with the negative sign:

$$
E_{\Sigma}=m_{g}^{\prime} c^{2}-E_{k}-U_{0}-W_{0}
$$


For the bodies that are only under influence of their proper gravitational and electromagnetic fields, the virial theorem is satisfied, according to which the absolute value of the potential energy of the field on the average is twice as much than the kinetic energy of substance:

$$
2 E_{k}+U_{0}+W_{0} \approx 0, \quad E_{t o t}=E_{k}+U_{0}+W_{0} \approx-E_{k} \approx \frac{U_{0}+W_{0}}{2},
$$

here $E_{t o t}$ is the total energy excluding the rest energy of the particles of the body.

Substituting (51) in (50) gives the approximate equality:

$$
E_{\Sigma}=m_{g}^{\prime} c^{2}-E_{t o t} \approx m_{g}^{\prime} c^{2}-\frac{U_{0}+W_{0}}{2} .
$$

We shall now consider the essence of the mass $m_{g}^{\prime}$ related to the substance mass excluding the contribution from the mass of the internal kinetic energy and the energy of macroscopic fields. The contributions in mass $m_{g}^{\prime}$ are made by the masses of various types of energy associated with atoms and molecules at the temperature near absolute zero: strong interaction, binding the substance of the elementary particles and retaining the nucleons in atomic nuclei; electromagnetic interaction of particles; the energy of motion of electrons in atoms; rotational energy of atoms and molecules; vibrational energy of atoms in molecules, etc. In Standard Model it is assumed that the strong interaction arises due to the action of the gluon field between the quarks located in the hadrons (mesons and baryons), and the strong interaction between leptons is absent.

There is also a hypothesis that the strong interaction is a manifestation of strong gravitation at the level of elementary particles and atoms [9]. Since gravitation has two components, in the form of the field of acceleration $G$ and the torsion field $\Omega$, the stability of nucleons in nuclei can be described as the balance of forces from the attraction of the nucleons to each other due to $G$, and the repulsion of nucleons due to the torsion field $\Omega$ [3]. The same idea is applied to describe the structure and the stability of a number of hadrons, considered as the composition of nucleons and mesons [4]. Strong gravitation differs from the ordinary gravitation by replacing of the gravitational constant $\gamma$ by the constant of strong gravitation $\Gamma$, and acts between all particles, including leptons. The estimation of the quantity $\Gamma$ can be obtained from the balance of four forces acting on the electron in the hydrogen atom: 1 . The force of electric attraction between the electron and the atomic nucleus. 2. The force of electric repulsion of the charged substance of the electron from itself (the electron is represented as a cloud around the nucleus). 3 . The centripetal force from the rotation of the electron around the nucleus. 4 . The attraction of the electron to the nucleus under the influence of strong gravitation. These forces are approximately equal to each other, so the relations for the forces of attraction from strong gravitation and the electric force are satisfied [5]:

$$
-\frac{\Gamma M_{p} M_{e}}{R_{e}^{2}}=-\frac{e^{2}}{4 \pi \varepsilon_{0} R_{e}^{2}}, \quad \Gamma=\frac{e^{2}}{4 \pi \varepsilon_{0} M_{p} M_{e}}=1.514 \cdot 10^{29} \mathrm{~m}^{3} \cdot \mathrm{kg}^{-1} \cdot \mathrm{c}^{-2},
$$

where $M_{p}$ and $M_{e}$ - the mass of proton and electron, respectively,

$R_{e}$ - the radius of rotation of the electron cloud,

$e$ - the elementary electric charge as the proton charge equal to the absolute value of the negative charge of electron,

$\varepsilon_{0}-$ the vacuum permittivity.

Another way to estimate $\Gamma$ is based on the theory of similarity of matter levels and the use of coefficients of similarity. These coefficients are defined as follows: $\Phi=1.62 \cdot 10^{57}$ - coefficient of similarity by mass (the ratio of the mass of neutron star to the proton mass); $P=1.4 \cdot 10^{19}$ - the 
coefficient of similarity by size (the ratio of the radius of neutron star to the proton radius); $S=0.23$ - the coefficient of similarity by speed (the ratio of the characteristic speed of the particles of neutron star to the speed of light as the typical speed of the proton substance). For strong gravitational constant a formula is obtained: $\Gamma=\gamma \frac{\Phi}{P S^{2}}$, where degrees of similarity coefficients correspond to the dimension of gravitational constant $\gamma$ according to the dimension theory.

If we understand the strong interaction as the result of strong gravitation, the main contribution to the proton rest energy should be made by the positive kinetic energy of its substance and the negative energy of the strong gravitation (the electrical energy of the proton can be neglected due to its smallness). The sum of these energies gives the total energy of the proton, and due to the virial theorem (51) this sum of energies is approximately equal to half of the energy of strong gravitation. Since the energy of the strong gravitation is negative, then the total energy of the proton is negative too. The total energy of the proton up to the sign can be regarded as the binding energy of its substance; the binding energy equals to the work that should be done to spread the substance to infinity so that there total energy of the substance (potential and kinetic) should be equal to zero. According to its meaning, the positive proton rest energy must be equal to the binding energy or the absolute value of the total energy of the proton. This gives the equality between the rest energy and the absolute value of half of the energy of strong gravitation:

$$
M_{p} c^{2}=\frac{k \Gamma M_{p}^{2}}{2 R_{p}}
$$

where $k=0.6$ for the case if the proton was uniform density ball with the radius $R_{p}$.

If we substitute (53) in (54), we obtain another equation, which allows estimating the radius of the proton:

$$
M_{e} c^{2}=\frac{k e^{2}}{8 \pi \varepsilon_{0} R_{p}}, \quad \quad R_{p}=\frac{k e^{2}}{8 \pi \varepsilon_{0} M_{e} c^{2}}=\frac{k r_{0}}{2}
$$

where $r_{0}$ is the classical electron radius.

In self-consistent model of the proton [10] we find that in (54) the radius of the proton is $R_{p}=8.73 \cdot 10^{-16} \mathrm{~m}$, and the coefficient $k=0.62$ due to a small increase in the density of substance in the center of the proton. At the same time, in the assumption that positive charge is distributed over the volume of proton similar to the mass distribution and the maximum angular frequency of the proton rotation is limited by the condition of its integrity in the field of strong gravitation, we can find the magnetic moment of the proton as a result of rotation of the charged substance:

$$
P_{m}=\delta e \sqrt{\Gamma M_{p} R_{p}}
$$

where $P_{m}=1.41 \cdot 10^{-26} \mathrm{~J} / \mathrm{T}$ is the magnetic moment of the proton,

$\delta=0.1875$ (in the case of the uniform density and the charge of proton it should be $\delta=0.2$ ).

Strong gravitational constant (53) explains not only the energy (54) and the magnetic moment (55) of the proton, but also gives an estimate of the constant of interaction between two nucleons by the strong gravitation:

$$
\alpha_{p p}=\frac{\beta \Gamma M_{p}^{2}}{\hbar c}=13.4 \beta
$$


where $\beta=0.26$ for the interaction of two nucleons, and it tends to 1 for particles with lower density of substance, $\hbar$ - Dirac constant, $c$ - speed of light.

For the energy of interaction of two nucleons, by means of pseudoscalar pion-nucleon interaction, located at a distance $r$ from each other, and for the corresponding constant of interaction at low energies, the following formulas hold:

$$
U_{s}=-\frac{g_{\pi N}^{2}}{4 \pi r} \exp \left(-\frac{M_{\pi} c r}{\hbar}\right), \quad \alpha_{\pi N}=\frac{g_{\pi N}^{2}}{4 \pi \hbar c} \approx 14.6
$$

where $g_{\pi N}$ is the effective charge of strong interaction, $M_{\pi}$ is the mass of the pion.

As we can see, the interaction constant $\alpha_{p p}$ is close to the constant $\alpha_{\pi N}$ of pseudoscalar pionnucleon strong interaction.

The fact that the rest energy of the proton is associated with strong gravitation, also follows from the modernized Fatio-Le Sage theory of gravitation [11]. In this theory, based on the absorption of the fluxes of gravitons in the substance of bodies with transfer of the momentum of gravitons to the substance, the exact formula for Newton's gravitational force (the law of inverse squares) is derived; the energy density of the flux of gravitons $\left(4 \cdot 10^{34} \mathrm{~J} / \mathrm{m}^{3}\right)$, the cross section of their interaction with the substance $\left(7 \cdot 10^{-50} \mathrm{~m}^{2}\right)$ and other parameters are deduced.

In the theory of infinite hierarchical nesting of matter [3], [5] it is shown that at each main level of matter the corresponding type of gravitation appears: there is a strong gravitation at the level of elementary particles, but at the level of stars it is the ordinary gravitation. The gravitation reaches a maximum in the densest objects - in nucleons and in neutron stars. In the substance of the earth's density the range of strong gravitation is less than a meter, and at such sizes of bodies strong gravitation is replaced by the ordinary gravitation. This corresponds to the fact that the masses and the sizes of objects at different levels of matter increase exponentially, and the point of replacing of the strong gravitation by the ordinary gravitation lies near the middle of the range of masses from nucleons to the stars on the axis of the masses on the logarithmic scale.

The fluxes of gravitons, which consist of particles similar to neutrinos, photons and charged particles, are considered the cause of gravitation and electric forces in the modernized Fatio-Le Sage theory. These fluxes of gravitons generated by the substance of lower levels of matter, control the bodies with the help of gravitational and electromagnetic forces and create massive objects at higher levels of matter. These objects, in turn, at certain stages of their evolution radiate portions of neutrinos, photons and charged particles that become the basis of other fluxes of gravitons, are already acting at higher levels of matter. So the fields and the massive objects mutually generate each other at different levels of matter.

In the above picture the rest energy of proton (54) is approximately equal to the absolute value of the total energy of the proton in its proper field of strong gravitation (for increased accuracy we should also take into account the electromagnetic energy of the proton), and the energy $m_{g}^{\prime} c^{2}$ in (52) consists of the rest energy of nucleons and electrons of the substance of the body, with the addition of the energy of their gravitational and electromagnetic interactions inside the substance and the mechanical motion in atoms and molecules. Consequently, the energy $m_{g}^{\prime} c^{2}$ of the body, taking into account the virial theorem (51) can be reduced to the half of the absolute value of the sum of the energy of strong gravitation $U_{0 g}$ and electromagnetic energy $W_{0 g}$ of the nucleons, electrons, atoms and molecules involved in formation of the binding energy. As a result, the total energy of the substance and the field of the body at rest instead of (52) can be written down as follows:

$$
E_{\Sigma} \approx-\frac{U_{0 g}+W_{0 g}}{2}-\frac{U_{0}+W_{0}}{2}
$$


To understand the meaning of energy $E_{\Sigma}$ better, we shall consider the energy balance in the process of merging of substance, with formation of elementary particles at the beginning, passing then to confluence of the elementary particles into atoms and finally in the formation of a body of many atoms. Initially, the substance is motionless at infinity and its parts do not interact with each other, so that total energy of the system is zero (we do not consider here the rest energy of substance in its condition when it is fragmented and was not yet included into the composition of the elementary particles). If the substance will draw together under the influence of strong then ordinary gravitation, the negative energy $U$ of gravitational field and the positive kinetic energy $E_{k}$ of motion of substance will appear, and due to the energy conservation law the total energy should not change, remaining equal to zero. In the energy balance it is necessary to take into account the electromagnetic energy $W$ and the energy $E_{r}$ leaving the system due to the emission of field quanta such as photons and neutrinos:

$$
E_{r}+E_{k}+U+W=0, \quad E_{r}=-\left(E_{k}+U+W\right)=-E_{\text {Tot }}=-\frac{U+W}{2} .
$$

In (57) the virial theorem (51) is used for the components of the total energy $E_{T o t}$ of the system. According to (57), the energy $E_{r}$ of the emission that left the system equal up to a sign to the total energy $E_{T o t}$, i.e. the energy of emission $E_{r}$ equals the binding energy of the system. By comparing (57) and (56) we now see that the total energy $E_{\Sigma}$ of substance and of the field of the body at rest is the same as the energy extracted from the body by different emission during the formation of the body. As a rule in the energy $E_{\Sigma}$ only those components are taken into account that are associated with formation of elementary particles, atoms and macroscopic molecular substance; and the binding energies of the particles of which the substance of elementary particles is built are not taken into account and are assumed to be constant. Heating the body due to gravitation according to (57) and (56) leads to an increase of body energy $E_{\Sigma}$. This conclusion is based on the fact that although the internal kinetic energy of the body $E_{k}$ is part of (57) with the negative sign, but the change of the potential energy $U+W$ by the virial theorem compensates the contribution of the energy $E_{k}$. An example is the star, which is heated and accelerates its rotation during compression by gravitation, and the absolute value of the gravitational energy of the star increases.

According to (56), the total energy $E_{\Sigma}$ of the body at rest, which is used in formulas (47) for calculation of the energy and the momentum of the moving body, consists mainly of the energies of two fundamental fields - gravitational and electromagnetic, responsible for the integrity of the particles of the body and for the composition of the body of the individual particles. In this case, the strong interaction between the particles is taken into account by the energy of strong gravitation $U_{0 g}$ and the electromagnetic energy $W_{0 g}$.

As for the weak interaction it is assumed to be the result of transformation of substance, which was for a long time under the influence of the fundamental fields. An example is the long-term evolution of a star massive enough to form a neutron star in a supernova outburst, when the neutrino burst is emitted with the energy of about the total energy of the star (the gravitational energy of the substance compression into a small-sized neutron star is converted into the energy of neutrinos, the energy of photon emission, the kinetic energy and the heating of the expelled shell). At the level of elementary particles, this corresponds to the process of formation of a neutron with the emission of neutrino.

If the body at rest in the weak interaction emits (absorbs by the body) neutrinos, photons and other particles, it leads to a change of the total energy $E_{\Sigma}$ of the body. In general, the energy $E_{\Sigma}$ of the body is the function of time and speed with which the separate particles or units of substances are emitted from the body or absorbed by it. Due to the laws of conservation of energy and momentum, if some particles bring into the system the energy and momentum, then after some time 
they are distributed in the system and according to virial theorem they can be taken into account through the energy and the momentum of the fundamental fields. Therefore, we can state that according to (56), the source of the total energy of the body, and of its mass $M_{\Sigma}=M$ as the measure of inertia are the gravitational and electromagnetic fields associated with the mass and charge (as well as the currents) in the substance. In Fatio-Le Sage theory of gravitation it is supposed that the fields associated with the mass and the charges are the consequence of the interaction of the substance and the charges with the fluxes of gravitons and tiny charged particles that penetrate the space. If we define the total mass of the body in the form $M_{\Sigma}=\frac{E_{\Sigma}}{c^{2}}$, then (47) is as follows:

$$
E=\frac{M_{\Sigma} c^{2}}{\sqrt{1-V^{2} / c^{2}}}, \quad \quad \boldsymbol{P}=\frac{M_{\Sigma} \boldsymbol{V}}{\sqrt{1-V^{2} / c^{2}}}
$$

\section{Conclusions}

Equations (58) look exactly the same as (1) for a small test particle. However, the body mass $M_{\Sigma}$ in (58) takes fully into account the field energies, whereas for the mass $m$ of a small particle in (1) it is only expected. The appearance in the mass $M_{\Sigma}$ of the contribution from the energy of fields has occurred because we have used the energy of mutual interaction of many small particles in a massive body. Hence, by induction, we should suppose that not only the mass of body, but the mass of any isolated small particle should be determined taking into account the contribution from the energy of proper fundamental fields of the particle. The described concept of mass in the covariant theory of gravitation (CTG) is confirmed by the analysis of the Hamiltonian [12] and of the Lagrangian in the principle of least action [13].

We should note the difference between the results of CTG and general theory of relativity (GTR) with respect to mass and energy. In CTG the mass of the uniform spherical body at rest with the radius $R$ is expressed by formulas (46) - (49):

$$
M=m^{\prime}-\frac{U_{0}+W_{0}}{c^{2}}=m^{\prime}+\frac{3 \gamma M^{2}}{5 R c^{2}}-\frac{3 q^{2}}{20 \pi \varepsilon_{0} R c^{2}},
$$

where the mass $m^{\prime}$ sets the mass of the body parts, excluding the potential energy of the fields. As a result the relativistic and gravitational mass $M$ of the body by combining the body parts into a whole increases due to the energy of the gravitation field $U_{0}$, and decreases due to the electric energy $W_{0}$.

In GTR the mass of the body $M$ in the weak-field approximation, according to (6) is determined by summing up all the energies and then dividing the result by the square of the speed of light:

$$
M=m+\frac{U_{0}+W_{0}}{c^{2}}=m-\frac{3 \gamma M^{2}}{5 R c^{2}}+\frac{3 q^{2}}{20 \pi \varepsilon_{0} R c^{2}} .
$$

In contrast to (59) in (60) not the mass $m^{\prime}$ but the mass $m$ is used, which is included in the formula for the rest energy and the Lagrangian. As it was shown in [12], for three masses associated with the body, the following relation holds: $m^{\prime}<M<m$. At the same time $m-M \approx M-m^{\prime}$, which also follows from (59) and (60). In our opinion, the reason of difference between (59) and (60) is associated with different positions of the two theories: in CTG there is explicit stress-energy tensor of the gravitational field, included in the Lagrangian and contributing to the spacetime metric and the energy-momentum of the considered system. This allows us to define all the three masses $m^{\prime}, M, m$ and to find their meaning, and the mass $m^{\prime}$ is associated with the cosmological constant in the equation for the metric of the system. In GTR the principle of equivalence is used instead of 
this, the gravitational field is reduced to the metric field, and correspondingly, the energy and the momentum do not form tensor and can be found only indirectly, through the spacetime metric. In GTR, only two masses, $M$ and $m$, have the meaning.

The equality $m-M \approx M-m^{\prime}$ means that the relativistic gravitational mass $M$ of the body is in the middle between the masses $m^{\prime}$ and $m$, and differs from them by the absolute value of the potential energy of fields. This implies consistency of positions CTG and GTR, as these theories determine the mass and energy from different standpoints.

\section{References}

1. Misner, Charles W.; Kip. S. Thorne \& John A. Wheeler (1973), Gravitation, W. H. Freeman, ISBN 0-7167-0344-0.

2. Okun L. B. Photons, Clocks, Gravity and the Concept of Mass. Nucl. Phys. B (Proc. Suppl.). Vol. 110, pp. 151-155 (2002).

3. Fedosin S.G. Fizicheskie teorii i beskonechnaia vlozhennost' materii. - Perm, 2009. - 844 p. ISBN 978-59901951-1-0.

4. Comments to the book: Fedosin S.G. Fizicheskie teorii i beskonechnaia vlozhennost' materii. - Perm, 2009, 844 pages, Tabl. 21, Pic. 41, Ref. 289. ISBN 978-5-9901951-1-0 (in Russian).

5. Fedosin S.G. Fizika i filosofiia podobiia: ot preonov do metagalaktik. - Perm, 1999. - 544 p. Tabl. 66, Pic. 93, Ref. 377. ISBN 5-8131-0012-1.

6. Heaviside, Oliver. Electromagnetic waves, the propagation of potential, and the electromagnetic effects of a moving charge, Electrical papers, Vol. 2, pp. 490-499 (1888/1894).

7. Fedosin S.G. Mass, Momentum and Energy of Gravitational Field. Journal of Vectorial Relativity, Vol. 3, No. 3, pp. 30-35 (2008).

8. Fedosin S.G. The Principle of Proportionality of Mass and Energy: New Version. Caspian Journal of Applied Sciences Research, Vol. 1, No 13, pp. 1-15 (2012).

9. Sivaram C. and Sinha K.P. Strong gravity, black holes, and hadrons. Physical Review D, Vol. 16, Issue 6, pp. 1975-1978 (1977).

10. Fedosin S.G. The radius of the proton in the self-consistent model. Hadronic Journal, Vol. 35, No. 4, pp. 349-363 (2012).

11. Fedosin S.G. Model of Gravitational Interaction in the Concept of Gravitons. Journal of Vectorial Relativity, Vol. 4, No. 1, pp. 1-24 (2009).

12. Fedosin S.G. The Hamiltonian in Covariant Theory of Gravitation. Advances in Natural Science, Vol. 5, No. 4, pp. 55-75 (2012).

13. Fedosin S.G. The Principle of Least Action in Covariant Theory of Gravitation. Hadronic Journal, Vol. 35, No. 1, pp. 35-70 (2012). 